\title{
ER stress boosts respiration
}

PERK-elF2a-
ATF4 axis ...
enables cells
to maintain
a state of
metabolic
fitness under
glucose
deprivation

In glucose-abundant conditions glycolysis supplies sufficient ATP for cell metabolism. However, when glucose availability is limited, cells require the more efficient mitochondrial oxidative phosphorylation (OXPHOS) for energy production. How mitochondrial metabolism adapts to nutrient availability is poorly understood. Balsa et al. now show that glucose deprivation induces endoplasmic reticulum (ER) stress, which enhances OXPHOS by promoting the assembly of mitochondrial respiratory complexes into supercomplexes.

OXPHOS is driven by respiratory complexes in the inner mitochondrial membrane (IMM). These complexes are known to assemble into larger supercomplexes, but their exact function remains elusive. Using human and mouse cell lines, the authors established that glucose deprivation increased the formation of these supercomplexes. This was accompanied by an increase in the density of mitochondrial cristae IMM folds that harbour respiratory supercomplexes and support their assembly and stability and enhanced mitochondrial

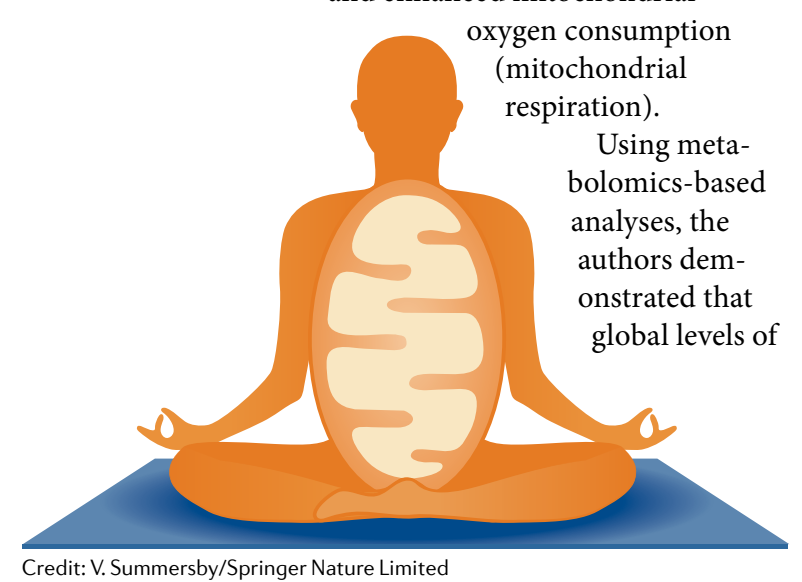

protein glycosylation were reduced in cells deprived of glucose and this defect correlated with the induction of ER stress. Moreover, inhibition of glycosylation by tunicamycin treatment, which induces ER stress, increased mitochondrial respiration, respiratory supercomplex assembly and cristae density in the presence of glucose. Similar changes were observed upon induction of ER stress by brefeldin A, which blocks ER-Golgi transport. Thus, ER responds to glucose deprivation, and the ensuing ER stress promotes respiratory supercomplex assembly.

A key mediator of ER stress is protein kinase R-like ER kinase (PERK). Loss of PERK, or inhibition of a PERK target, eukaryotic initiation factor $2 \alpha$ (eIF2 $\alpha$ ), interfered with the assembly of respiratory supercomplexes in glucose-free conditions. Conversely, PERK activation was sufficient to increase supercomplex formation and promoted mitochondrial respiration both in cultured cells and in mouse liver tissue.

Supercomplex assembly is driven by supercomplex assembly factor 1 (SCAF1, also known as COX7A2L). Transcription of SCAF1 was induced in cells cultured in glucose-free medium, which depended on PERK and eIF2 $\alpha$ activity. SCAF1 depletion reduced the number of supercomplexes, mitochondrial oxygen consumption and cellular ATP levels in the absence of glucose, which led to reduced cell proliferation. These defects were also observed following depletion of activating transcription factor 4 (ATF4), which is a PERK-eIF2 $\alpha$ effector. These data indicate that the PERK-eIF2 $\alpha-A T F 4$ axis directly promotes respiratory supercomplex assembly and OXPHOS, which enables cells to maintain a state of metabolic fitness under glucose deprivation. Further experiments revealed that these respiratory chain adaptations require concomitant remodelling of the IMM with increased cristae assembly downstream of ER stress.

Complex I dysfunction is among the most common causes of mitochondrial disease. Human cells that harbour disease-associated complex I mutations cannot adapt to glucose deprivation and undergo cell death upon glucose removal. Notably, cells with mutations that interfere with complex I stability could be rescued from cell death in the absence of glucose by pre-treatment with tunicamycin (before glucose removal). Thus, activation of ER stress has the ability to counteract complex I instability in the context of mitochondrial disease.

Therefore, more efficient ATP production by mitochondria through increased assembly of respiratory supercomplexes downstream of ER stress could provide a robust mechanism to adapt to various stresses, including starvation and protein stress. These results provide novel insights into the functional communication between the ER and mitochondria. They also demonstrate that the assembly of respiratory supercomplexes has a functional role in supporting mitochondrial respiration.

Paulina Strzyz

ORIGINAL ARTICLE Balsa, E. et al. ER and nutrient stress promote assembly of respiratory chain supercomplexes through the PERK-elF2a axis. Mol. Cell https://doi.org/10.1016/j.molcel. 2019.03.031 (2019) 